\title{
Comunicación, incidencia política y planificación
}

\author{
Washington Uranga \\ Universidad Nacional de La Plata / Universidad de Buenos Aires / Universidad Nacional de San luis \\ wuranga@wuranga.com.ar
}

\begin{abstract}
Resumen
La planificación de y desde la comunicación solo puede pensarse y entenderse como una práctica política de incidencia sobre escenarios sociales diversos y atravesados por la conflictividad propia de las luchas por el poder de las que participan los actores en el territorio. Estas acciones exigen la elaboración de estrategias comunicacionales que, partiendo de análisis situacionales, identifiquen actores y nudos críticos, para dar respuestas complejas al margen de toda simplificación. La comunicación necesita de un proyecto que le otorgue sentido y que pueda ser comunicado.
\end{abstract}

Palabras clave: comunicación, política, planificación, incidencia

\section{Resumo}

O planejamento da e desde a comunicação só pode ser pensado e entendido como uma prática política de incidência em cenários sociais diversos e perpassados pelo conflito inerente às lutas pelo poder das quais os atores do território participam.

Essas ações requerem a elaboração de estratégias de comunicação que, com base na análise situacional, identifiquem atores e nós críticos para fornecer respostas complexas, além de qualquer simplificação. A comunicação precisa de um projeto que Ihe dê significado e que possa ser comunicado.

Palavras chaves: Comunicação, política, planejamento, incidência

Aunque pueda resultar hasta paradójico en un texto que pretende debatir sobre comunicación, incidencia política y planificación, resulta esencial iniciar la reflexión problematizando en el escenario de nuestras prácticas el sentido íntegro del concepto de comunicación poniendo el eje en el sujeto como actor relacional y protagonista de las transformaciones sociales. Especialmente cuando el predominio de los discursos mediáticos y los debates acerca de su influencia en el escenario de la política, relega a un segundo plano del sentido básico de la 
REVCOM | ISSN 2451-7836 | Año 4, \#8 | mayo de 2019 | Dossier temático | e002

comunicación como hecho relacional que tiene al ser humano como centro y protagonista de la misma. De allí la importancia de, sin negar otras miradas y enfoques, reafirmar la perspectiva que entiende la comunicación como hecho socio-político-cultural, atinente a todo lo social, vinculado al hacer y a la forma del hacer. Como bien lo señala el maestro Jesús Martín-Barbero:

La comunicación es el 'hecho social total' por excelencia como esfuerzo integrador y como experiencia concreta, o mejor aún, como espacio totalizador de lo social. Decir que la comunicación es acción es integrarla a la praxis que dialectiza ${ }^{1}$, diferencia y referencia, construcción y realidad, relación y creación (Martin-Barbero 2018:105).

En la misma línea argumental Adalid Contreras sostiene que

es preciso rechazar la concepción instrumental de la comunicación reducida a medios o a información; se debe renunciar a la idea mitológica de la comunicación persuasiva publicitariamente hasta el infinito; tenemos que volver a la característica más elemental de la comunicación: communicare ${ }^{2}$, o poner en común, ponernos en relación, para seguir interrogándonos sobre el mundo, problematizando la vida, descongelando voluntades, sentipensando propositivamente las realidades complejas, buscando colectivamente respuestas, definiendo caminos compartidos, conquistando espacios esperanzadores, reencantando la vida buena en armonía y retomando permanente y organizadamente el futuro. (Contreras Baspineiro 2018: 284).

Esta comprensión de la comunicación y el acento en la complejidad, sumada a la perspectiva integral de derechos (que incluye el derecho a la comunicación) redefine, por un lado, el quehacer y el rol del/de la comunicador/a en la sociedad, pero también reposiciona a la comunicación en el marco de la política y de las decisiones políticas. No se puede, a nuestro juicio, pensar la comunicación al margen de la política, de las relaciones de poder, de la construcción de modelo de sociedad. Por eso coincidimos en que "ser comunicador es, ante todo, un estilo de hacer" (Massoni, 2011: 32) y en que el comunicador es "un profesional con capacidad para interpelar la dinámica social, para operar crítica y valorativamente en su dimensión comunicativa" y cuya tarea de reconocimiento de la diversidad sociocultural, "es indagar los posibles puntos de articulación de las diferencias en función de intereses y necesidades de grupos sociales que se reconocen como distintos, para operar desde allí en relación a un objetivo de transformación cognitiva macrosocial" (Massoni, 2011: 62).

1 El destacado es del autor.

2 El destacado es del autor. 


\section{ReVcom \\ REVISTA CIENTIFICA DE LA REDCOM}

REVCOM | ISSN 2451-7836 | Año 4, \#8 | mayo de 2019 | Dossier temático | e002

Al mismo tiempo y basado en similares argumentos se puede afirmar que el objeto de estudio de los comunicadores son las prácticas sociales y no exclusivamente los medios o los productos comunicacionales. Nos referimos a prácticas sociales comprendidas como la acción colectiva, organizada o no, consciente o no, en la que intervienen actores diversos poniendo en juego sus saberes, intereses y poderes, generando formas de interacción que adquieren significación y que provocan consecuencias sociales, económicas, culturales y políticas, configurando los procesos históricos.

Nuestra preocupación se centra entonces en los procesos comunicacionales, entendidos como interacciones entre sujetos en el ámbito histórico de la vida cotidiana, lugar en el cual se producen, intercambian y negocian formas simbólicas y se generan sentidos atravesados por intereses y formas de poder.

Las prácticas comunicativas en la sociedad son muchas y muy diversas. No existe un modelo dentro del cual se las pueda encasillar, porque responden a la esencia misma del ser humano como sujeto libre, de condición humana compleja y básicamente social. Tales prácticas se recrean por los hábitos propios de la cultura, que van generando nuevas ritualidades, por el desarrollo de técnicas y tecnologías de comunicación, y por el entrelazamiento y la interacción de ambos campos en el ámbito de la vida cotidiana de las personas. Pueden describirse también como el ser y actuar de los sujetos en la historia. Las personas van tejiendo y generando los procesos comunicacionales en toda su complejidad. La cultura es la malla densa que se produce en el intercambio comunicativo, pero que a la vez es sustento de los sujetos y sus procesos a los que contiene y les da sentido.

Como nos pasa en otros órdenes de la vida también en la comunicación es difícil escapar a la oscilación pendular que nos lleva a enfatizar un aspecto en desmedro de otro, siendo que ninguno de ellos es ni menos importante ni menos significativo. Tanto en la práctica comunicacional como en las reflexiones que se hacen sobre ella resulta difícil encontrar el punto cierto. Que no es equilibrio - una instancia que puede ser sinónimo de algo inerte, sin movimiento, sin dinamismossino una respuesta para la tensión entre los aspectos relacionantes de la comunicación más vinculada a la cultura, a la construcción de identidades y que expresan la construcción de sentidos entre los sujetos, por una parte, y aquellas dimensiones sociales y políticas que atraviesan el escenario de la industria y de los medios masivos, y cercana a la economía política de la comunicación, por otra. En todo, por donde se lo quiera mirar, ambos aspectos están indisolublemente ligados a la política. 


\section{ReVcom \\ REVISTA CIENTIFICA DE LA REDCOM}

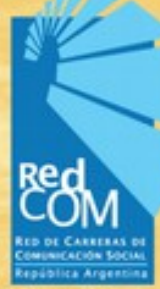

REVCOM | ISSN 2451-7836 | Año 4, \#8 | mayo de 2019 | Dossier temático | e002

En todos los casos es necesario no perder de vista el sentido constituyente de la comunicación, en tanto vincularidad entre personas, entre actores. También porque asistimos y somos coprotagonistas de un escenario político cultural en el que en lugar de puentes se construyen trincheras, y antes que propiciar el diálogo y el encuentro la preocupación está centrada en el enfrentamiento y en la eliminación de reales o imaginarios adversarios, en no pocos casos concebidos directamente como enemigos. $\mathrm{Y}$ en el que la comunicación, sus estrategias, tecnologías e instrumentos, son utilizados con el mismo propósito.

Frente este panorama perversamente binario que obstaculiza el análisis y hace difícil el discernimiento diremos, acompañamos las palabras del colombiano Omar Rincón referidas al periodismo pero aplicables a la comunicación en general, para sostener que

lo que hay que comenzar a pensar es cómo hacemos para que el papel de la comunicación sea conectar a los diferentes, como conectamos diversos pensamientos y ponemos en diálogo a la sociedad. Ese es el objetivo fundamental de la comunicación y lo que está haciendo la comunicación hoy, sobre todo en el periodismo informativo, es dividiendo. No le interesa juntar sino dividir. Entonces creo que hay que pensar en el papel del periodismo, que es construir lazos entre la sociedad, la conversación pública entre todos ${ }^{3}$.

Para comprender la comunicación y trabajar desde allí al servicio de la construcción colectiva hay que abandonar la lógica binaria y dualista que divide el mundo entre "buenos" y "amigos" (los que piensan lo mismo que el enunciador) y "malos" o "enemigos" (los que discrepan con aquel). Para ello

es preciso admitir que los 'buenos' no están en un solo bando, porque aún siendo nosotros mismos parte de los 'buenos' hay también 'buenos' en muchos otros lugares y, muy probablemente, en nuestro propio espacio nos estemos codeando también con una cantidad importante de 'malos'. También porque lo 'bueno' y lo 'malo' cohabita inevitablemente en el interior de cada sujeto, de cada colectivo, de cada situación (Uranga 2016: 67).

Planificar la y desde comunicación es intervenir en los procesos sociales. Por lo tanto, planificar la comunicación es hacer política porque siempre existe vocación y decisión de incidencia. Pero, siguiendo el razonamiento anterior, esta tarea de planificar no puede aportarse

3 Entrevista a Omar Rincón en el diario El Tribuno (Salta, 14 de junio de 2018). Consulta realizada el 7 de marzo de 2019 en https://www.eltribuno.com/salta/nota/2018-6-14-12-24-0-omar-rincon-se-requierebuen-periodismo-porque-abunda-la-posverdad. 
REVCOM | ISSN 2451-7836 | Año 4, \#8 | mayo de 2019 | Dossier temático | e002

del propósito fundamental de generar puentes, apuntalar el diálogo en y desde la diferencia para facilitar la amistad y el encuentro social.

Para ello y para que la comunicación sea vehículo y sostén de la transformación necesitamos pensarla en la tensión permanente entre la producción de sentidos en el espacio de lo micro, de lo próximo y lo cercano, pero atendiendo de manera simultánea a la comunicación masiva, a las industrias culturales y los desarrollos tecnológico infocomunicacionales. La vida cotidiana de los sujetos es el lugar de síntesis cultural ineludible de todos estos vectores. Comunicar la persona, las personas, desde sus identidades en relación, con el escenario de los medios masivos y de la cultura. Bucear en las experiencias de comunicación popular, comunitaria y alternativa para aprender de ellas, de sus estrategias pero también de sus estéticas. Trabajar en el espacio de los medios y la cultura masiva para reconocer allí a los sujetos, actores y protagonistas de la historia. Trazar estrategias de incidencia a través de las redes sociales digitales. No son mundos distintos, son todos ellos ámbitos en diálogo, también en tensión y en competencia, dentro del mismo escenario de producción colectiva de sentidos y, por ende, de conocimientos. Es parte de la complejidad que nos atraviesa y a la que hay que prestar permanente atención.

Comunicar para la transformación, planificar la incidencia desde la comunicación, implica pensar al sujeto comunicador como un protagonista de la historia social. Un sujeto que se construye y constituye atravesado simultáneamente por sus entornos más próximos (el grupo de referencia y de pertenencia, el trabajo, el grupo familiar, sus relaciones, etc.) y por su inserción en el espacio social, político y de la cultura masiva. La identidad político cultural de un actor no depende de un solo espacio de actuación, por más que uno pueda tener más incidencia que otro en la configuración del sujeto comunicador. Siempre hay una amalgama compleja de influencias que se cruzan y una inevitable hibridación a la que contribuyen el sujeto mismo, los contextos y las interrelaciones y en la cual los énfasis dependen de circunstancias muy diversas. Todo eso hace a la construcción de matrices culturales que van configurando a las personas y a los grupos entendidos como actores sociales.

Mirar la complejidad y desde la complejidad es también dejar atrás las miradas fragmentarias tanto respecto de la política como de la comunicación. Retomando lo antes señalado, afirmamos que pensar y analizar los procesos comunicacionales desde una perspectiva de transformación demanda recoger lo mejor de nuestras tradiciones en comunicación popular, alternativa, comunitaria, poniéndolas en conjunción con aquellas configuraciones que atiendan a la comunicación como industria y como cultura masiva y del entretenimiento. Lo comunitario y popular 
REVCOM | ISSN 2451-7836 | Año 4, \#8 | mayo de 2019 | Dossier temático | e002

en la comunicación no puede ser contrario y opuesto a la cultura masiva y comercial. Los productos culturales de los medios masivos no están condenados, por ser tales, a ser contradictorios con un proyecto de cambio. Y las redes sociales digitales, sus lenguajes y potencialidades tienen que ser integradas en la planificación de proyectos de comunicación con perspectiva transformadora.

Aquí está uno de nuestros desafíos y una de las paradojas que tenemos que descifrar. Hacer comunicación con perspectiva de cambio social, planificar la comunicación y desde la comunicación a partir de una mirada integral que rescate la centralidad del sujeto presente en la comunicación popular asumiendo sus aspiraciones y, al mismo tiempo, incorporar todos los recursos y las posibilidades que brindan los desarrollos tecnológicos infocomunicacionales y la producción masiva de bienes culturales. Todo demanda organización, planificación y calidad en la ejecución y en la producción para lograr impacto y sustentabilidad -económica, política y cultural- para alcanzar los objetivos deseados y que, en consecuencia, la propuesta tenga sentido.

Lo anterior lleva también a decir que las comunicadoras y los comunicadores somos parte integrante -con derechos y responsabilidades- de las sociedades que habitamos. Estamos conformados y constituidos sobre la base de la misma escala de valores y vivimos las mismas contradicciones y tensiones de las que coparticipan el resto de los actores sociales. En esa línea de razonamiento, se nos debería exigir similar nivel de protagonismo y responsabilidad social que al resto de los actores sociales. Ni más ni menos. Y ubicarnos en pie de igualdad con todos ellos para no convertirnos -por la vía que sea- en "árbitros" -mucho menos en jueces- de las acciones de terceros.

Los comunicadores y las comunicadoras estamos llamados a ser "promotores y facilitadores del diálogo público en el espacio público, en gestores de otras palabras y otros discursos, pero también y necesariamente, en artesanos de un escenario diverso y polifacético" (Uranga op. cit.: 229). Parafraseando a M. Foucault podríamos decir que no somos nosotros comunicadores y comunicadoras a quienes corresponde "concientizar" a los oprimidos, a los equivocados o sencillamente a los "malos". Se trata, en cambio, de penetrar en el silencio, en los silencios que abundan en el escenario social y en sus actores, para romper la opacidad del secreto y forzar la red discursiva. De esta manera, planificar la comunicación puede entenderse como una acción destinada a "dar vida a lo colectivo incorporando manos provistas de habilidades diferentes para diseñar prácticas distintas y voces que permitan configurar la imprescindible polifonía de lo social" y eso requiere entender al comunicador "como facilitador de otras maneras de ser y actuar y de diferentes formas de construirlas en el espacio público" (Uranga op. cit.: 230) 
REVCOM | ISSN 2451-7836 | Año 4, \#8 | mayo de 2019 | Dossier temático | e002

\section{Planificar desde la comunicación es intervenir en lo social}

La planificación desde y de la comunicación no puede ser entendida sino como una iniciativa compleja de intervención en las prácticas sociales. Una acción que se lleva a cabo usando la comunicación como categoría central y como eje de análisis, pero que reconoce la complejidad de las prácticas $\mathrm{y}$, que por lo tanto, necesita acudir a los aportes de otras disciplinas para desentrañar, comprender, analizar el territorio entendido como escenario de actuación.

La planificación, comprendida como intervención, puede ser leída desde tres dimensiones inseparables y muchas veces difícilmente reconocibles de manera autónoma en las acciones de las personas y los grupos: producción de conocimiento, acción política y proceso comunicacional. Estas tres dimensiones son constitutivas de la práctica de los actores sociales y, en consecuencia, de los comunicadores que tienen por vocación incidir en los procesos de cambio social.

La planificación en tanto proceso abordaje de un territorio-escenario con intenciones de transformación, constituye por sí misma una manera de conocer a partir del intercambio comunicativo con otros actores, con la cultura y con el ámbito de actuación. Quien interviene pone en juego su concepción acerca del sujeto y del mundo, pero también abre al diálogo entre sus propias concepciones y aquellas presentes en el territorio dando lugar a la dimensión política de su práctica.

Conocemos cuando, utilizando nuestras categorías analíticas desarrollamos metodologías y construimos herramientas para la intervención y mediante ellas accionamos en el territorio independientemente de que el objetivo manifiesto o el pretexto sea el análisis o el cambio social. La práctica de la intervención encierra en sí misma una fase del conocimiento y supone además un proceso de interacción de saberes entre la diversidad de los actores presentes en el escenario de actuación leído como territorio.

Dado que todo proceso de intervención implica también una investigación para la acción, para el cambio, la teoría nos permite hacer inteligibles las prácticas que analizamos, interpretarlas para luego orientar las acciones. Pero a la vez se generan nuevas conceptualizaciones como consecuencia de la dialéctica reflexión-acción, acción-reflexión. Todo proceso de intervención puede ser vivido como una situación de aprendizaje, como una experiencia educativa que nace de la práctica y de las preguntas y respuestas que de ella se derivan, tanto para quienes realizan la intervención (en este caso los/las comunicadores/as) como para quienes son actores directos en el territorio. Esta experiencia educativa es también un modo de conocimiento. 
REVCOM | ISSN 2451-7836 | Año 4, \#8 | mayo de 2019 | Dossier temático | e002

Pero la intervención es además una instancia de mutuas incidencias entre saberes que pueden denominarse de distinto orden y alimentados desde prácticas y recorridos diferentes, que interactúan entre sí para generar nuevas categorías y criterios que permitan modificar los preexistentes o generar otros absolutamente novedosos. Hay en ello una clara perspectiva política que encierra modos de comprender el mundo y sus formas organizativas. También una metodología para la acción. En ese sentido la intervención es acción política y lucha simbólica por el poder.

"Entendemos por incidencia una acción sostenida en el tiempo con objetivo de transformación social y perspectiva de derecho que busca influir y generar discusión pública sobre un determinado tema". (Uranga y Thompson, coord.: 2016: 53). Y tal incidencia puede darse por lo menos en los siguientes niveles: darle visibilidad a un tema; sensibilizar sobre un tema; instalar agenda sobre un tema; participar en la definición de políticas públicas. Cada uno de estos niveles supone diferentes grados de compromiso de los actores, habilidades y capacidades distintas. Pueden ser comprendidos también como grados de desarrollo en relación al tema que se está abordando.

Una organización social desarrolla habilidades y capacidades organizacionales para la incidencia cuando:

- tiene claridad respecto de su misión en torno al tema/derecho que le preocupa y sus miembros poseen objetivos compartidos:

- la experiencia en el terreno ha sido problematizada a partir de la lectura crítica del contexto de intervención (el escenario) y del tema que los convoca (el derecho pretende garantizar);

- genera acciones para dar a conocer lo que hace y dar visibilidad al derecho en diversos ámbitos del espacio público;

- se propone la sensibilización de otros actores clave en torno a ese tema y su instalación en la agenda política;

- busca la participación activa en la toma de decisiones para el diseño de las políticas públicas específicas en relación con el tema que le preocupa siendo un interlocutor habitual del Estado sobre el tema de su competencia.

No es necesario pensar que la incidencia solo se logra mediante el desarrollo y la puesta en práctica de todas estas habilidades y capacidades. En la mayoría de los casos no existe conciencia organizacional acerca de las mismas y tampoco pueden distinguirse todas ellas yde manera precisa como aquí se las presenta desde el punto de vista analítico. 
REVCOM | ISSN 2451-7836 | Año 4, \#8 | mayo de 2019 | Dossier temático | e002

\section{Estrategias para la acción}

La planificación también puede ser pensada como la articulación inteligente, eficaz y productiva de estrategias que se suman para alcanzar un objetivo de cambio.

Niveles y capacidades se combinan para dar lugar a las estrategias, entendidas como curso de acción, conscientes y proyectados, que orientan la voluntad de transformación puesta de manifiesto por los actores o por las organizaciones. La estrategia suele traducirse en una orientación o en un conjunto de directrices que orientan y organizan la acción colectiva.

La estrategia es una forma de hacer o de generar criterios para el hacer. También como múltiples caminos complementarios que elegimos recorrer para alcanzar determinados propósitos o escenarios propuestos a modos de imagen de futuro posible y deseable. Pero, desde una mirada más epistemológica, se puede decir que la estrategia es una forma de percibir el mundo que permite una determinada ubicación para el hacer, para el cambio, para la acción transformadora. Toda estrategia es, al mismo tiempo, una manera de entender las relaciones entre los sujetos (comunicación que nos habla de vincularidades y entramados que determinan modos de ser y actuar), una forma de mediación que genera siempre novedad (la "en-acción"), que supone asumir lo "fluido" (el cambio constante y continuo) y lo complejo (multicausalidad y multiplicidad de consecuencias) y cuya resolución demanda de saberes multidisciplinares (Pérez y Massoni: 2009).

Las estrategias para la incidencia asoman, en consecuencia, como respuestas posibles a los nudos críticos o desafíos generados a partir de la convergencia de situaciones problemáticas y se configuran como articulaciones complejas que operan de manera interconectada y complementaria sobre distintas dimensiones. Es la manera como las organizaciones sociales (también las personas) producen acciones articuladas entre sí para obtener la vigencia plena de un determinado derecho o alcanzar un propósito manifiesto.

Por ese motivo la estrategia se ubica en el plano de la inteligencia de la acción, constituyéndose en el lugar donde se entrecruzan las relaciones, los sentidos, los sueños, los imaginarios y los productos propios de los medios en el marco de los sistemas que los contienen. Es también la relación entre el sujeto, como actor y protagonista de la historia, y la cultura con la que dialoga que, al mismo tiempo, lo atraviesa y lo constituye.

Así es como la estrategia en comunicación empieza a estar cada vez más asociada a poner en marcha procesos de concertación social para la movilización mediante la creación de consensos 
REVCOM | ISSN 2451-7836 | Año 4, \#8 | mayo de 2019 | Dossier temático | e002

y la negociación de propósitos colectivos. Para lograrlo es preciso comprender cómo se comunica la sociedad y cómo articula imaginarios potentes de transformación de su realidad, reconociendo la diferencia y el conflicto y construyendo escenarios de encuentro, de concertación y de participación (Jaramillo López: 2012).

Del mismo modo la planificación de la comunicación estratégica no puede quedar limitada a la producción y circulación de información. Tiene que estar orientada a promover intercambios, a suscitar diálogos y conversaciones de modo tal que llegue a constituirse en una instancia decisiva, clave, en los procesos de organización, construcción y cambio social. Tomando en cuenta siempre que este proceso está regado de conflictos, contradicciones y negociaciones que deben ser contemplados y atendidos por el diseño estratégico de la comunicación.

Jaramillo López (op. cit. p. 8) sostiene que "el mejor aporte reside en la construcción de escenarios de encuentro, concertación y participación para la creación de consensos y propósitos colectivos". Este es el camino por habría que transitar. Se puede decir también que las estrategias son la forma de traducir en iniciativas la voluntad política y cultural de generar el cambio, que tiende a acompañar y fortalecer un proyecto de desarrollo, un entramado social, político, organizacional o de políticas públicas.

\section{Planificar el encuentro en la diversidad}

Frente a las dificultades que nos presenta un escenario social y políticamente conflictivo y multiculturalmente diverso, ensayar una propuesta comunicacional reconociendo la diferencia y la diversidad, basada en el diálogo y el intercambio entre los actores, puede ser entendida a veces como ingenua y otra veces como facilista o solución mágica. Ante ello hay que reafirmar que la comunicación no es una herramienta fantástica que hace desaparecer la conflictividad propia de los procesos sociales. Muy por el contrario: los conflictos y las diferencias anidan en los procesos comunicacionales. En consecuencia, planificar la comunicación demanda rigurosas tareas de análisis situacional, identificación y mapeo de actores y relaciones, para comprender todo en sus contextos y en sus procesos.

Política y metodológicamente lo anterior es el punto de partida inevitable para, reconociendo las diferencias, poner a dialogar a los actores desde sus identidades y desde las matrices culturales que los constituyen. Es la manera de encaminar diálogos comunicacionales constructivos, al margen de los discursos vacíos, para construir alternativas concretas y propuestas que, reconociendo las diferencias, construya instancias superadoras de encuentro 
REVCOM | ISSN 2451-7836 | Año 4, \#8 | mayo de 2019 | Dossier temático | e002

en la diversidad. Es una tarea repetidamente declamada y escasamente ejecutada, que necesita más acciones que la pongan en práctica, menos agentes publicitarios y más militantes de la causa más importante: el ser humano gozando de derechos humanos en plenitud.

Frente a la crisis, a la violencia, ante la incertidumbre, pero también en el camino de la búsqueda de mejor calidad de vida, los hombres y las mujeres exteriorizan sus necesidades de encuentro, de intercambio. Aunque esto no lleve siempre a las coincidencias. Diálogo es comunicación, es construcción colectiva, es espacio de intercambio. Muchas veces de concertación y otras tantas de lucha, de debate. El diálogo es necesario, es materia prima para generar el cambio, pero demanda que se construyan las condiciones para el mismo. Y, sobre todo, que no se pretenda caer en la simplificación de que diálogo es sinónimo de acuerdos, de coincidencias, de falta de tensiones. El diálogo es una actitud, una disposición y no un resultado. Se da siempre en el contexto de una sociedad atravesada por las tensiones, las diferencias y los juegos de poder.

Ese diálogo se concreta en el marco de la conflictividad social. Es un proceso turbulento y complejo que pone en juego la lucha de identidades. Allí quedan en evidencia las pretensiones de dominio de las distintas comunidades, grupos y actores sociales. La mayor parte de las veces, este es también un proceso inevitablemente violento; en lo simbólico seguramente y, en no pocos casos, también en lo físico.

Para no caer en una mirada ingenua es preciso advertir que este proceso de encuentro en la diversidad no puede analizarse al margen de las relaciones de poder, de las dominaciones económicas, políticas y sociales. Son los actores sociales, los hombres y las mujeres, quienes sufren la dominación. Y la dominación es integral, atraviesa las prácticas sociales, las culturas. En todo el territorio y en sus escenarios podemos reconocer simultáneamente marcas que nos hablan de dominaciones de todo tipo en los actores y en los sujetos, que a la vez que padecen y son víctimas de la dominación son también ejecutores de prácticas opresivas y contrarias a una perspectiva de derechos.

En medio de este escenario conflictivo tenemos que planificar la comunicación con perspectiva de incidencia y sentido político. Pero las estrategias de comunicación no son apenas bengalas, señales luminosas o fuegos de artificio como pretenden algunos titiriteros del marketing político y comunicacional. Son herramientas que pueden ayudar a potenciar y hasta convertir en exitoso un proyecto -así sea mediocre- si logra identificar fibras o imaginarios sensibles de los públicos y construir un relato en torno a los mismos. Es necesario tener un 
REVCOM | ISSN 2451-7836 | Año 4, \#8 | mayo de 2019 | Dossier temático | e002

proyecto para comunicar, un sentido que alimenta la acción. No se puede hacer comunicación desde la nada, si no hay nada que comunicar. No menos cierto es que en la actualidad las mejores iniciativas pueden fracasar por una inadecuada o ineficiente estrategia comunicacional que no acierte en apelar a los sentidos movilizadores de la ciudadanía.

Todo lo anterior para decir también que siendo indiscutible el tan reiterado argumento de la concentración de la propiedad de los medios en Argentina y en América Latina, también puede convertirse en un pretexto si, por ejemplo, no se atiende y no se saben leer múltiples manifestaciones comunicacionales presentes en el territorio para recogerlas como parte esencial del relato político pero, a su vez, para ensayarlas como estrategias comunicacionales complementarias con los medios -así sea escasos- de los que se disponga.

Habla la calle con sus manifestaciones, con los reclamos, con las demandas. Habla el trémulo murmullo del descontento en el tren, en el subte y en el colectivo. Grita el reclamo ante el abuso. Hay mensaje y relato en las concentraciones de las mujeres, en el verdurazo, en los ruidazos y cacerolazos, en las movilizaciones de los docentes. Hay mensaje hasta en el atronador silencio de las comunidades originarias que no pueden hacer oír su voz. También en la ausencia de las dirigencias ante los temas más acuciantes que deberían estar en la agenda de sus preocupaciones.

La política y la comunicación necesitan acercar el oído al territorio, escuchar a sus actores, atender sus voces, transitar sus senderos. Porque es allí donde están los contenidos y las estrategias y no apenas en los gabinetes de los especialistas y de los dirigentes, aunque éstos también sean necesarios. Porque siendo el escenario de los problemas y de los padecimientos, el territorio es al mismo tiempo el lugar de los sueños y de los deseos. Más allá de las quejas y la demandas y sin abandonarlas de ninguna manera, es preciso que afloren desde allí las imágenes de futuro para que, como bien lo señala Gastón Berger, desde ahora ese imaginario se convierta en "razón de ser del presente" (citado en Godet, 1995: 2). Sin ese pre requisito todo lo que se intente puede quedar vacío de sentido $\mathrm{y}$, por lo tanto, ineficiente e incapaz para dar respuestas políticas y comunicacionales que conmuevan y sean adecuadas a los desafíos actuales. 


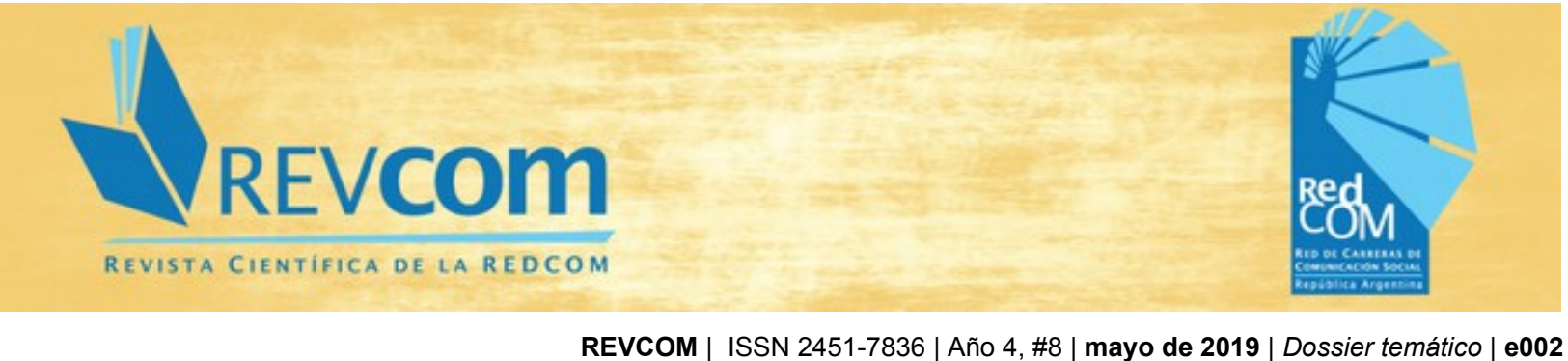

\section{BIBLIOGRAFÍA}

Contreras Baspineiro, A. (2018), Recordar el futuro. Planificación de la comunicación desde la comunicación. Neiva, Editorial Universidad Sucolombiana.

Godet, M. (1995). De la anticipación a la acción. Barcelona: Alfa omega.

Jaramillo López, J. (2012). "Propuesta general de comunicación pública". Strategy \& Management Busines

Review. vol 3 (2) pp. 1-17. Consultado el 14 de marzo de 2019 en http://www.exeedu.com/publishing.cl/strategy_manag_bus_rev/2012/Nol3/Nro2/1-SM17-11-full.pdf

Perez, R. y Massoni. S, (2009).Hacia una teoría general de la estrategia. Barcelona. Ariel Comunicación.

Martín-Barbero, J. (2003). De los medios a las mediaciones. Comunicación, cultura y hegemonía. Bogotá. Editorial Gilli,

Martín-Barbero, J. (2018). La palabra y la acción. Por una dialéctica de la liberación. Bogotá. Editorial Pontificia Universidad Javeriana.

Massoni, S. (2011). Comunicación estratégica. Comunicación para la innovación. Rosario. Homo Sapiens.

Massoni, S. (2013). Metodologías de la comunicación estratégica. Del inventario al encuentro sociocultural. Rosario. Homo Sapiens.

Morín, E. (2004). Introducción al pensamiento complejo. México, D. F.. Editorial Gedisa.

Uranga, W. (2016). Conocer, transformar, comunicar. Buenos Aires. Editora Patria Grande.

Uranga W. y Thompson H. (coord.). (2016). La incidencia como camino para la construcción de ciudadanía. Una propuesta para trabajar desde la comunicación. Buenos Aires. Editora Patria Grande. 